\title{
Pillules in Single-dose Container
}

National Cancer Institute

\section{Source}

National Cancer Institute. Pillules in Single-dose Container. NCI Thesaurus. Code C149759.

Medicinal product consisting of pillules presented in a single-dose container. 